\title{
Revisiting Appendicular Lump
}

\author{
Bhandari RS, 'Thakur DK, 'Lakhey PJ,' Singh KP' \\ 'Department of surgery, TU Teaching Hospital, Kathmandu, Nepal.
}

\section{ABSTRACT}

Introduction: Appendicular lump is a well known sequalae of acute appendicitis encountered in 2$6 \%$ of patients. Successful management of appendicular lump is controversial with different approaches. As many controversies are arising regarding management of appendicular lump. The aim of this study was to find out the outcome and evaluate possible need of changing our management strategy of appendicular lump.

Methods: A retrospective analysis of the patients managed with appendicular lump were done. All the patients admitted with diagnosis of appendicular lump and managed between, over two and half years, were included in the study. All age groups and both sex were included. Any patients whose diagnosis was changed after initial diagnosis of appendicular lump were excluded from the study.

Results: Total 75 patients had appendicular lump suggesting 10\% incidence. Age varied between 1183 years with nearly equal incidence in both sexes. Majority had onset of symptoms between 2 to 14 days with an average of 4 days. Average stay was 3 to 4 days. During study period, 12 (16\%) came with recurrence and $13(17 \%)$ cases came for elective appendectomy.

Conclusions: Based on our finding, it is not sufficient to change our classical management strategy of appendicular lump and suggests a need for long term prospective study in this very common clinical condition.

Key Words: appendicular lump, conservative management

Correspondence:

Dr. Ramesh Singh Bhandari

Department of surgery, TUTH

Maharaiguni, Kathmandu, Nepal.

Email: rameshbhandari2000@yahoomail.co.in

Phone: 9841270203 


\section{INTRODUCTION}

Acute appendicitis is one of the most common surgical conditions of the abdomen and appendicular lump being its sequalae is encountered in $2-6 \%$ of patients. ${ }^{1,2}$ Majority of the times appendicular lump resolves after conservative management ${ }^{1}$ or at times may get complicated necessitating urgent surgical management. Successful management of an appendicular lump is controversial with three general approaches. 'Classical management' involves initial conservative management followed by interval appendicectomy following resolution of symptoms. ${ }^{3}$ Recently, the need for interval appendicectomy has been questioned, with a number of authors adopting an entirely conservative approach without interval appendicectomy. ${ }^{4} \mathrm{~A}$ third approach involves performing immediate appendicectomy during the initial admission prior to resolution of the mass. ${ }^{5}$ In our hospital we are usually following classical conservative approach.

Following successful conservative management of appendicular lump, actual fate of this condition has not been studied in our set up. As many controversies are arising regarding management of appendicular lump, there are no actual data to support our management strategy that every patient with appendicular lump requires interval appendectomy. We do not have true incidence of recurrent appendicitis, complications following appendicular lump and elective appendectomies. So, in an attempt to find answers to these questions, this study has been conducted.

\section{METHOD}

A retrospective study the patients managed with appendicular lump was conducted in the Department of Surgery, Tribhuvan University Teaching Hospital from December, 2006 to June, 2008. Institutional approval was taken. All the patients admitted with diagnosis of appendicular lump and managed between, over two and half years, were included in the study. All age groups and both sex were included. Any patients whose diagnosis was changed after initial diagnosis of appendicular lump were excluded from the study.

All the patients with diagnosis of appendicular lump were managed with standard conservative approach of OchsnerSherren regimen and advised for elective appendectomy. Necessary interventions were done when necessary. Parameter studied were demographic data, duration of symptoms, length of hospital stay, complications, recurrence of appendicitis, rate of elective appendectomies and follow ups. Data were analyzed with SPSS 14.0 for windows software.

\section{RESULTS}

Total 75 patients of appendicular lump were included for analysis. Total cases of appendicitis, including appendicular lump, managed in our hospital during the study period were 758. So, total incidence of appendicular lump was $10 \%$. Age range varied between $11-83$ years and median age group was between $20-29$ years. Majority of the patient were between 21 to 40 years of age (table 1). Male and female ratio was 1.08: 1 (Table 2) suggesting almost equal incidence in both groups. Most of the patient had onset of symptoms between 2 days to 14 days with an average of 4 days in majority of the cases (table 4). Overall length of hospital stayed varied between 2- 10 days with an average stay of 3 to 4 days in most of the cases.

During conservative management, 5 (7\%) cases developed appendicular abscess and in three cases we could successfully manage it with ultrasound guided drainage while 2 cases required formal laparotomy, drainage and successful appendectomy at same setting. Except the prolonged hospital stay, all cases had successful recovery. During study period, $12(16 \%)$ cases returned to our hospital with repeat attack of acute appendicitis and they all underwent successful appendectomy. The time duration of recurrence of symptom varied between 4 weeks to 2 years with a median duration of 9 weeks. Similarly, $13(17 \%)$ cases returned to hospital for elective appendectomy with in duration of 6 weeks to 8months and all of them underwent successful appendectomy although with some difficulty in finding appendix in few cases. One case, 55yrs female, on follow up was found to have Caecal malignancy after thorough evaluation and she underwent right hemicolectomy. Total 47 cases i.e. $63 \%$ cases failed to come to follow up. We couldn't retrieve the actual cause of the lost follow ups.

Table 1. Age distribution

\begin{tabular}{ll}
\hline Age range & Frequency \\
\hline$\leq 20$ & 12 \\
$21-40$ & 39 \\
$41-60$ & 18 \\
$>60$ & 6
\end{tabular}

Table 2. Sex Distribution

\begin{tabular}{ll}
\hline Sex & Frequency \\
\hline male & 39 \\
female & 36 \\
total & 75 \\
\hline
\end{tabular}


Bhandari et al. Revisiting Appendicular Lump

Table 3. Duration of symptoms at presentration

\begin{tabular}{ll}
\hline Symptoms duration(days) & Frequency \\
\hline$\leq 2$ & 11 \\
$3-4$ & 32 \\
$5-6$ & 15 \\
$>6$ & 17 \\
\hline
\end{tabular}

\section{DISCUSSION}

Acute appendicitis is a very common cause of acute abdomen requiring surgical intervention. With prolongation of duration of symptoms, it usually forms appendicular lump which is an inflammatory mass composed of inflamed appendix, caecum, omentum, terminal ileum and at times sigmoid colon or tubes and ovaries in females. ${ }^{2}$ This has been attributed to a protective mechanism of body to prevent the spread of infection. In our study, we found that the incidence of the appendicular lump was approximately $10 \%$ and this is in comparable incidence of appendicular lump in other large centres where incidence has been found varying from 2 - $10 \%$ depending upon the literature. ${ }^{6}$

Most of the patients presented with appendicular lump were between third decades. However the age group varied from 11 yrs to 83 years suggesting any age group prone to develop lump but common in younger age groups. It has been said that the younger children do not have well formed omentum to form a lump while old age people have high risk of more rapid progression to complication like appendicular perforation peritonitis. ${ }^{7,8}$ Similarly, majority of the patients who presented with lump had symptoms between 3 to 4 days. However some even presented with symptoms for 14days. Usually when attack of appendicitis prolongs, body either tries to localize the infection by forming a lump or it gets complicated to abscess formation or generalized peritonitis. ${ }^{7}$

The success rate of initial conservative management varies between $76-97 \% .{ }^{9}$ In our study, out of total 75 cases of lump, $5(7 \%)$ developed abscess. Remaining patients were successfully managed by conservative management. So our success rate of conservative management was $93 \%$ comparable to other studies. As the practice at our institute is elective appendectomy following conservative management, every patient were advised for elective appendectomy. But only $13(17 \%)$ patients came for elective surgery and when asked majority of them wanted to have least chance of recurrence. Majority of the patients reported between 6 weeks to 8 months for elective surgery.

Total $47(63 \%)$ cases failed to come for follow up till the time we started analyzing the cases. Last case enrolled in our study was admitted 6 months prior to analyzing data. Actual cause for lost follow up could not be retrieved. Since this significant number of cases did not come for elective appendectomy, it aroused a valid question on our management strategy that does every patient really required the elective surgery? So, we did a literature search on this practical query. In our series, $16 \%$ of cases presented with recurrent appendicitis and majority of the patients presented with in 9 weeks of discharge.

A review article in 1993 by Ntecki et al reported a mean incidence of recurrent appendicitis in a meta-analysis of 329 patients managed conservatively as $13.7 \%(0-20 \%) . .^{10}$ Most recurrences occurred within the first two years. In a regional study, Kaminski et al identified 1012 patients over a 13 year period, with an appendix mass who had successful initial non operative treatment. "Eight hundred and sixty four patient did not have interval appendectomy. Thirty-nine of the 864 (5\%) had a recurrence at a mean follow up of 48 months. The mean time to recurrence was $10 \pm 15$ months. They performed multivariate analysis on these patients and concluded that the only factor associated with recurrence was male gender. They concluded that interval appendectomy was not justified as the vast majority $(95 \%)$ of patients successfully managed conservatively will not develop a recurrence and that a minority of patients who do recur will be admitted to hospital for less time than if they had had interval appendectomy.

Dixon et al reviewed the characteristics of 32 patients who had recurrence of symptoms following conservative management. ${ }^{12}$ Mean time to recurrence was five months following the initial episode. They compared the clinical characteristics of each patient at initial admission with the same characteristics at recurrence. They demonstrated that recurrent appendicitis was associated with milder clinical course. The recurrences were treated successfully with both operative and non- operative approaches and were not associated with any significant mortality and morbidity. They also compared clinical and demographic characteristics of the recurrence group to the non-recurrence group and found no significant risk factor for recurrence, including the severity of the initial presentation.

There is, therefore, good evidence, firstly, that the risk of recurrent acute appendicitis following successful conservative management is low; between $5 \%$ and $14 \%$. Secondly, in the minority of patients whose symptoms do recur, this usually occurs with in one year. Thirdly, recurrence of appendicitis following conservative management is usually associated with milder clinical course amenable to both operative and nonoperative approaches. Fourthly, there is no accurate method for predicting patients at risk recurrence.'

However, reasons justifying interval appendectomy is to exclude the possibility of an alternative pathology such as malignancy being misdiagnosed as an appendix mass. The incidence of misdiagnosing an appendix mass varies between $0-10 \%$. $^{10}$ Following non operative management of an appendix mass most authors consider further investigations as mandatory. ${ }^{4}$ The consequences of misdiagnosing an intra-abdominal malignancy as an appendix mass are serious. It is, therefore, essential to rigorously exclude other diagnosis with investigation in follow ups. In our series, the only misdiagnosis was 55 year old female with caecal carcinoma. After initial successful management, on follow up evaluation, malignancy was detected and she underwent right colectomy with successful outcome. Ceacal malignancy has been found in around 1-4\% of appendicular lump. ${ }^{9}$ In our study, it was $1.5 \%$.

Although majority of the centres worldwide, including ours, routinely follow classical conservative approach of OchsnerSherren regime for managing appendicular lump, different controversies are arising regarding its management. Advocates 
of immediate appendicectomy describe the advantages of avoiding the need for readmission for interval appendicectomy, and the exclusion of other pathologies masquerading as an appendix mass. ${ }^{5}$ Advocates of interval appendicectomy describe the advantages of avoiding recurrence of symptoms and the misdiagnosis of an appendix mass. ${ }^{13}$ They suggest interval appendicectomy is a less hazardous and challenging operation, compared with immediate appendicectomy during the initial admission. ${ }^{13}$ Proponents of an entirely conservative approach claim appendicectomy, whether interval or immediate, is unnecessary. ${ }^{14}$

None of these three approaches has gained universal acceptance. But reviewing different evidences from literature it seems logical to redefine our management strategy. As majority of our patients lost follow up, we could not stand strongly to support this. So, this small study has broadened our view and has suggested us to carry out a long term well documented prospective study to search strong evidence in modifying our management strategy of this very common surgical condition.

\section{CONCLUSIONS}

Reviewing evidences from literature, we found strong evidences against routine elective appendectomy following successful conservative management of appendicular lump. As majority of the patients in our study lost follow up, we could not derive any conclusion in support of this strategy. But from the available findings in our study and literature review, our view in managing appendicular lump has widened and urged us to carry out a long term prospective study in search of evidence in changing management strategy of this very common clinical condition.

\section{REFERENCES}

1. Jordan JS, Kovalcik PJ, Schwab CW. Appendicitis with a palpable mass. Ann Surg. 1981;193:227-9.

2. Arnbjornsson E. Management of Appendiceal Abscess. Curr Surg. 1984;41:4-9.

3. D.E. Deakin, I Ahmed. Interval appendectomy after resolution of adult inflammatory appendix mass- is it necessary? Surgeon. 2007;1:45-50.

4. Adalla SA. Appendiceal mass: Interval appendicectomy should not be the rule. Br J Clin Prac. 1996;50:168-9.

5. De U, Ghosh S. Acute appendectomy for appendicular mass: A study of 87 patients. Ceylon Med J. 2002; 47: 117-8.

6. Willemsen PJ, Hoorntje LE, Eddes EH, Ploeg RJ. The need for interval appendectomy after resolution of an appendiceal mass questioned. Dig Surg. 2002;19:216-20.

7. Haribhakti S, Appendicular diseases. Clinical GI surgery manual. 2008;1:457-62.

8. Hui TT, Major KM, Avital I. Outcome of elderly patients with appendicitis: effect of computed tomography and laparoscopy. Arch Surg. 2002;137:995-8.
9. Hung-Wen Lai, Che-Chuan Loong, Jen-Hwey Chiu, Gar-Yang Chau, Chew-Wun Wu, Wing-Yui Lui. Interval appendectomy after conservative treatment of an appendiceal mass. World J Surg. 2006; 30:352-7.

10. Nitecki S, Assalia A, Schein M. Contemporary management of the appendiceal mass. Br J Surg. 1993; 80: 18-20.

11. Kaminski A, Liu II, Applebaum H, Lee SL, Haigh PI. Routine interval appendectomy is not justified after initial nonoperative treatment of acute appendicitis. Arch Surg. 2005; 140: 897-901.

12. Dixon MR, Haukoos JS, Park IU, Oliak D, Kumar RR, Arnell TD, Stamos MJ. An assessment of the severity of recurrent appendicitis. Am J Surg. 2003;186:718-22.

13. Friedell ML, Perez-Izquierdo M. Is there a role for interval appendicectomy in the management of acute appendicitis? Am Surg. 2000;66:1158-62.

14. Tingstedt B, Bexe-Lindskog E, Ekelund M, Andersson R. Management of appendiceal masses. Eur J Surg. 2002; 168: 57982. 